\title{
Impact of the active area position in a nitride tunnel junction vertical-cavity surface-emitting laser on its emission characteristics
}

\author{
Patrycja ŚPIEWAK*, Michal WASIAK, Robert P. SARZAŁA \\ Institute of Physics, Lodz University of Technology, \\ Wólczańska 219, 90-924 Łódź, Poland \\ ${ }^{*}$ Corresponding author: patrycja.spiewak@edu.p.lodz.pl
}

\begin{abstract}
This paper presents results of numerical simulations of a nitride semiconductor vertical-cavity surface-emitting laser (VCSEL) with a tunnel junction. The modeled laser is based on a structure created at the University of California in Santa Barbara. The analysis concerns the impact of the position of laser's active area on the emitted power. Both small detunings from the standing wave anti-node, and positioning of the active area at different anti-nodes are considered.
\end{abstract}

Keywords: GaN, computer simulations, tunnel junction, VCSEL.

\section{Introduction}

Vertical-cavity surface-emitting lasers (VCSELs) have many advantages in comparison with edge-emitting lasers (EELs). Nowadays, VCSELs made of arsenide materials, emitting near-infrared radiation are successfully used. VCSELs fabricated from nitride materials with InGaN/GaN quantum-well (QW) active areas are not commercially available yet. The main advantages of VCSELs include: low threshold currents, relatively good parameters of the emitted beam and low price in mass production $[\underline{1}, \underline{2}]$.

The first nitride VCSELs operating at room temperature with continuous wave (CW) have been presented by groups from National Chio Tung Univeristy (NCTU) [ $\underline{3}]$ and Nichia Corporation [2]. However, the fabrication of GaN-based VCSELs is difficult. One of the main problem is the production of distributed Bragg reflectors (DBRs). The fabrication of DBRs from native materials is not easy due to the lattice mismatch between different nitride compounds ( $\mathrm{AlN}, \mathrm{GaN}$ and $\mathrm{InN}$ ). Another solution is to use dielectric mirrors, which from the technological point of view are easier to fabricate. In addition, the refractive index contrast possible to obtain between different dielectric materials is much higher than in nitride materials, so fewer layers of dielectric DBRs are needed to fabricate effective mirrors. On the other hand, dielectric materials have low thermal conductivity. Therefore, the use of dielectric DBRs is associated with a great challenge connected with removal of the heat from the structure. 
The group from the University of California in Santa Barbara (UCSB) develops nitride VCSELs with dual dielectric DBRs [ $\underline{4}, \underline{5}]$. A VCSEL presented in [4] will be investigated in this paper. This structure has a tunnel junction (TJ) and implantation as a current confinement. The main advantage of structures with a TJ is the fact, that p-type material is used only as a thin layer between the active region and the TJ. P-type GaN has a very low electrical conductivity, and higher optical absorption, compared to $\mathrm{n}-\mathrm{GaN}$, so reducing the thickness of $\mathrm{p}-\mathrm{GaN}$ can be beneficial. What is more, in a structure with a TJ both (top and bottom) electric contacts are deposited on n-type material, resulting in significantly lower contact resistance than in the structures with a p-type contact [6]. The other approach to obtain a current confinement is the use of a high-conductivity, semitransparent ITO (indium tin oxide) contact on the p-type side instead of a TJ. ITO VCSELs have a thick layer of $\mathrm{p}-\mathrm{GaN}$ with low electrical conductivity, therefore the use of ITO electrode is necessary to obtain uniform current distribution in the laser's aperture. The first nitride VCSELs had ITO contacts and nowadays the technology for fabricating these structures is more developed than the TJ VCSEL technology. This can be one of the reasons why VCSELs with ITO achieve the highest output powers. The ITO VCSEL presented recently by the Faculty of Science and Technology from Meijo University and R\&D Laboratories, Stanley Electric Co. achieved almost $16 \mathrm{~mW}$ output power in $\mathrm{CW}$ operation [7].

The laser's performance is strongly influenced by the precision of the fabrication of the device. For example, an imprecise positioning of the active area relative to the anti-node of the standing wave in the resonator may cause no laser action. In the case of a hybrid structure (a resonator with dielectric DBRs), the resonator has to be etched in order to obtain the designed thickness, however this process cannot be controlled as precisely as epitaxial growth. As a result, the distance from the DBRs to the elements such as the active region or the tunnel junction in real structures may be different than designed due to imperfections in the technological processes.

GaN-based VCSELs often have much longer resonators than VCSELs made of arsenide materials. Long resonators are needed because of the very low thermal conductivity of the DBRs (dielectric or AlInN/GaN). In a long resonator the lateral heat transport is more efficient, which can greatly reduce the thermal resistance of the device. Usually, because of the difficult control over the position of the active region relative to the DBR, in GaN-based VCSELs the active area is located at a short distance from one of the DBRs. In this paper, we investigate the impact of small deviations in the position of the active region as well as possible advantages resulting from positioning of the active region much deeper in the resonator.

\section{Simulated structure}

The simulated structure is based on the structure fabricated at UCSB [4]. The simulated VCSEL is designed to emit at $405 \mathrm{~nm}$. The original structure has a relatively long resonator of 22.5 $\lambda$. In Fig. 1, a scheme of the analyzed VCSEL is shown. The structure 


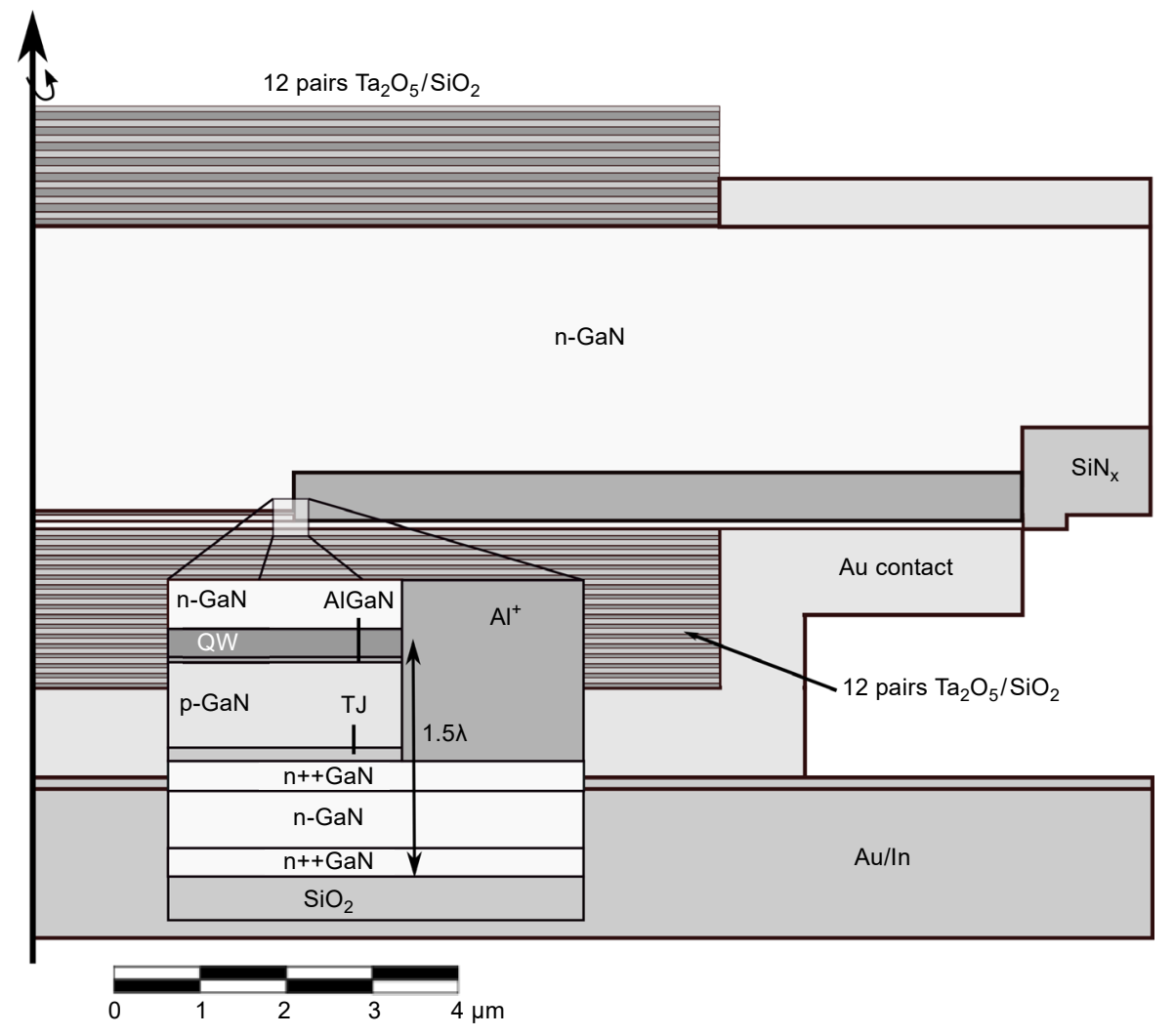

Fig. 1. Scheme of the analyzed nitride TJ VCSEL.

has two dielectric DBRs made of $\mathrm{Ta}_{2} \mathrm{O}_{5}$ and $\mathrm{SiO}_{2}$. The top and the bottom DBRs contain 12 and 16 pairs, respectively. The bottom laser contact has the form of a gold ring surrounding the bottom DBR. The active area of the laser consists of a double quantum well (DQW). The InGaN QWs are $14 \mathrm{~nm}$ wide, while the GaN barrier is $1 \mathrm{~nm}$ wide. Below the active area there is a $\mathrm{p}-\mathrm{GaN}$ layer and a tunnel junction. The TJ consists of $14 \mathrm{~nm}$ of $\mathrm{p}^{++} \mathrm{GaN}$ and $32 \mathrm{~nm}$ of $\mathrm{n}^{++} \mathrm{GaN}$. The absorption of the $\mathrm{p}^{++} \mathrm{GaN}$ is assumed to be $2000 \mathrm{~cm}^{-1}$, while in the $\mathrm{n}^{++} \mathrm{GaN}$ the absorption is $280 \mathrm{~cm}^{-1}$. The active area of the laser is at a distance of $1.5 \lambda$ from the bottom DBR (see the magnification in Fig. 1). The outer area of the laser is implanted by ions of aluminium. This implantation forms a high-resistive area that confines the current flowing through the laser.

The simulations were performed using computer models created in Photonics Group at the Lodz University of Technology. These computer models allow for calculation of the thermal, electrical, gain and optical phenomena occurring in the laser in the self -consistent way. Details of the computer models can be found in [ㅈ] and the material parameters used in the simulations presented in this paper are described in $[\underline{9}, \underline{10}]$. The validity of our models has been confirmed by experimental results [11-13]. 


\section{Results}

\subsection{Tuning of the active area position}

The analyzed structure is designed in such a way that the $29 \mathrm{~nm}$-thick active area is placed at the anti-node of the standing wave. In our simulations, the active area is shifted $+5 /-9 \mathrm{~nm}$ (upwards or downwards). Figure 2 shows the distribution of the electric field of the optical mode for +5 and $-9 \mathrm{~nm}$ shift of the active region in the areas near the active region. Because of the high absorption, the TJ is placed in such a way that the node of the standing wave is in the middle of the $\mathrm{p}^{++} \mathrm{GaN}$ (Fig. 2). The absorption of $\mathrm{n}-\mathrm{GaN}$ forming the greatest part of the resonator (see Fig. 1) is assumed to be $23.5 \mathrm{~cm}^{-1}$ [9]. In further analysis, we will also consider other values of absorption in this material. More details of the optical, electrical and thermal parameters of the analyzed structure can be found in $[\underline{10}]$.

Figure 3 presents $L-I$ (light-current) characteristics for different active area shifts. It shows that a shift as small as $+2 \mathrm{~nm}$ results in a slight increase in laser output power.

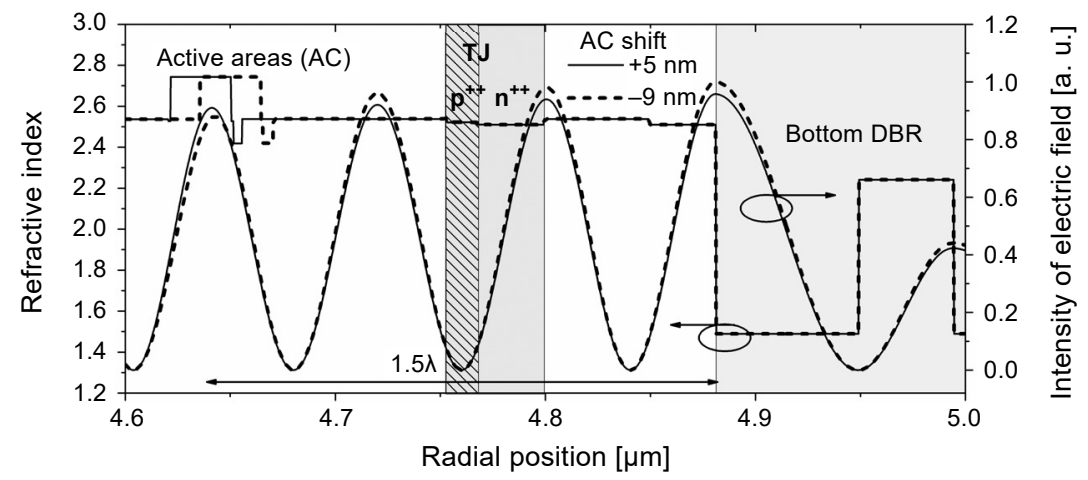

Fig. 2. Distribution of the electric fields of the electromagnetic radiation in the resonator near the active areas and tunnel junction (TJ) for active area shifts of $5 \mathrm{~nm}$ upwards and $9 \mathrm{~nm}$ downwards.

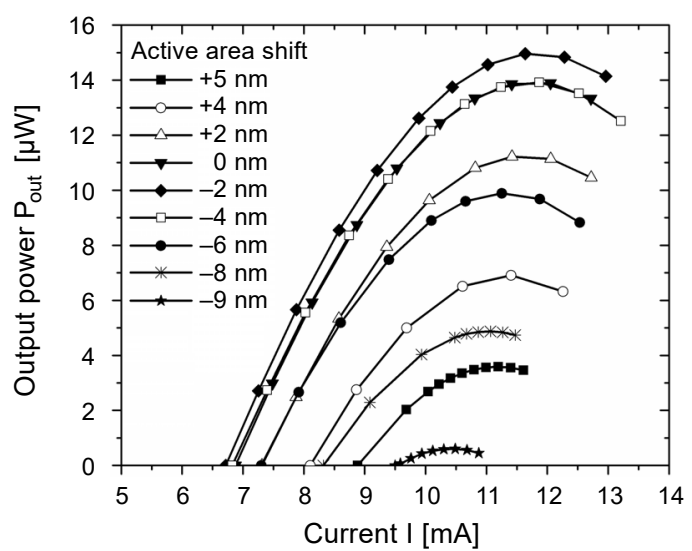

Fig. 3. Output power characteristics $(L-I)$ for lasers with different shifts of the active area. 
This is probably caused by the temperature-induced change of refractive indices of the laser layers. The power emitted by the laser depends on the material gain in the active area and the confinement factor $\Gamma$ defined by the following formula:

$$
\Gamma=\frac{\int_{\mathrm{AC}} n_{\mathrm{R}}^{2} E^{2} \mathrm{~d} x \mathrm{~d} y \mathrm{~d} z}{\int_{\operatorname{Res}} n_{\mathrm{R}}^{2} E^{2} \mathrm{~d} x \mathrm{~d} y \mathrm{~d} z}
$$

where, $n_{\mathrm{R}}$ is the local refractive index, $E$ is the amplitude of the electric field of the optical field, AC denotes the active region, while Res is the whole laser.

Figure 4 presents a graph of the confinement factor as a function of the shift of the active area. According to results from Fig. 4, the best modal gain is obtained when the active area is shifted by $-2 \mathrm{~nm}$ ( $2 \mathrm{~nm}$ downwards). These results are consistent with

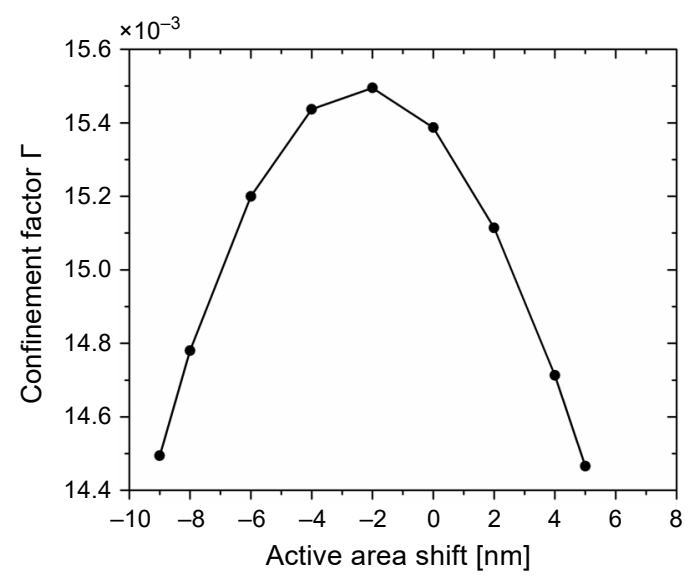

Fig. 4. Confinement factor $\Gamma$ as a function of the active area shift.

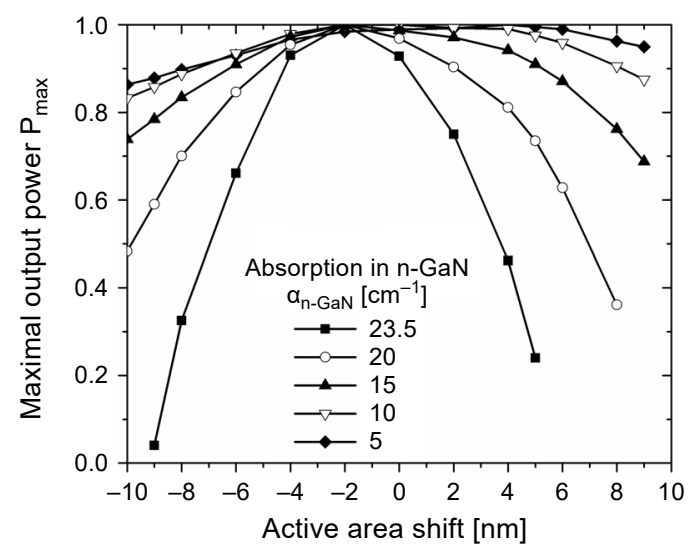

Fig. 5. Maximal output powers as functions of the active area shift for different values of absorption in $\mathrm{n}-\mathrm{GaN}$ resonator layer, the initial position is $0 \mathrm{~nm}$. 
the results from Fig. 3. The laser achieves the highest output powers when $\Gamma$ is the highest.

The maximal values of the emitted power depend, among others, on the absorption in the materials constituting the laser. In the considered VCSEL with the resonator of $22.5 \lambda$, most of the resonator is made of $\mathrm{n}-\mathrm{GaN}$ (placed between the active area and the top DBR). Therefore, the influence of the absorption in $\mathrm{n}-\mathrm{GaN}$ on the laser's performance was analyzed. The value of absorption in real structures depends on many factors. The values reported in [년 $\underline{16}$ ] are between 10 and $30 \mathrm{~cm}^{-1}$, but in a recent paper [17] the authors say that the absorption in $\mathrm{n}-\mathrm{GaN}$ can be as low as $1 \mathrm{~cm}^{-1}$. In our analysis we consider absorption in the range from 5 to $23.5 \mathrm{~cm}^{-1}$. Figure 5 presents the maximum values of the power $P_{\max }$ as a function of the active region shift for different values of the absorption. The values of emitted powers are presented relative to the highest value obtained in each case. According to the results in Fig. 5, when the absorption of the $\mathrm{n}-\mathrm{GaN}$ layer decreases then the shift of the active area has a lower impact on the maximal output power emitted by the laser.

\subsection{Active area at the center of the resonator}

In nitride VCSELs, even though the resonators are many- $\lambda$-thick, the active area is located close to one of the DBRs. In lasers without a TJ it allows for decreasing the thickness of the high-resistivity p-type GaN layer. In a laser with a TJ, the active area and the TJ can be placed in an arbitrary position without increasing the laser's resistance, however there is another reason why the active area should be located near one of the DBRs. Because the thickness of the resonator can be controlled with a limited accuracy, the active area will not be perfectly aligned with an anti-node of the standing wave. The higher the distance between the active area and the nearest DBR is, the bigger the misalignment will be. This is due to the fact that the anti-node of the standing wave is generated at the border between the resonator and the DBR. Even if the laser is designed to emit a certain wavelength, in the real resonator the wavelength will be slightly different. The misalignment $\Delta m$ between the successive anti-nodes of the ac-

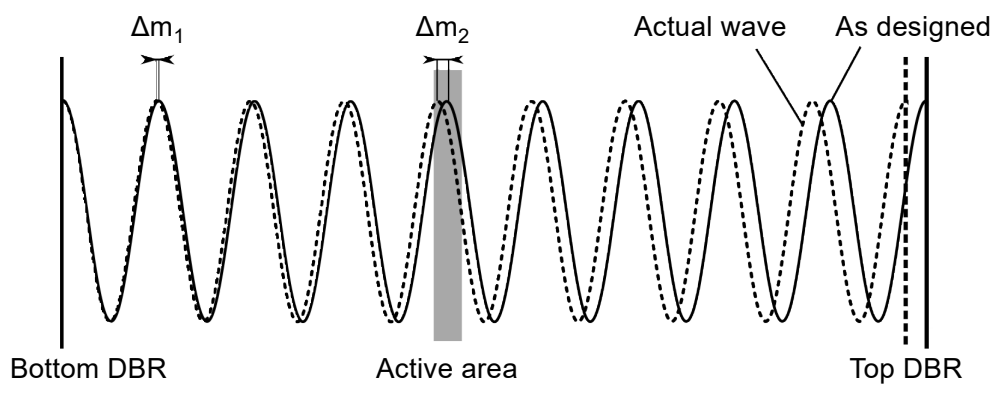

Fig. 6. Comparison of distributions of standing waves in two resonators of similar widths. Numbers $\Delta m_{i}$ denote the distances between the corresponding anti-nodes. 
tual standing wave and the anti-nodes in the designed resonator increase with the distance from the nearest DBR, i.e. $\Delta m_{2}>\Delta m_{1}$ (see Fig. 6). This could be one of the reasons why in the existing TJ nitride VCSELs the active area is placed close to the bottom DBR $[\underline{4}, \underline{18}]$. In principle, however, it is possible, in a TJ nitride VCSEL, to have the active region located at the center of the resonator. In what follows we will show certain benefits resulting from moving the active region deeper into the resonator.

Figure 7 presents thermal maps for two different distances $d_{\mathrm{AC}}$ between the active area and the bottom DBR, namely $1.5 \lambda$ and $11.5 \lambda$, at threshold conditions. For $d_{\mathrm{AC}}=$ $=11.5 \lambda$ the active region is placed at the anti-node of the standing wave in the middle

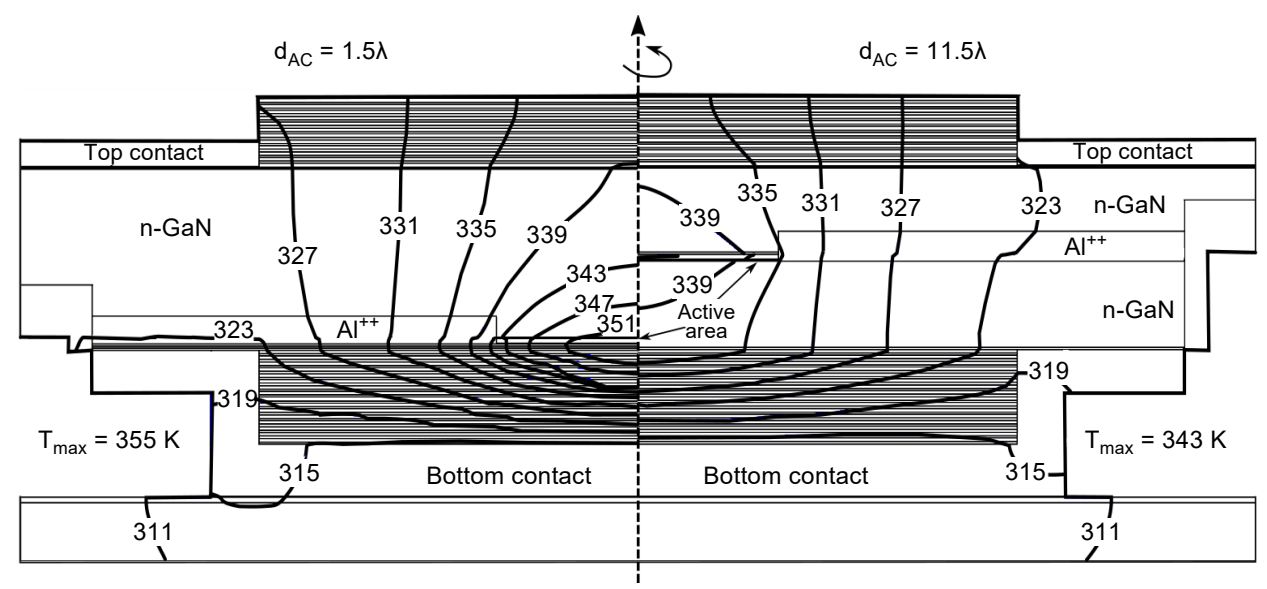

Fig. 7. Thermal maps of analyzed TJ VCSELs with the distance from the bottom DBR to the active area of $1.5 \lambda$ (left) and $11.5 \lambda$ (right). The results are for threshold conditions.

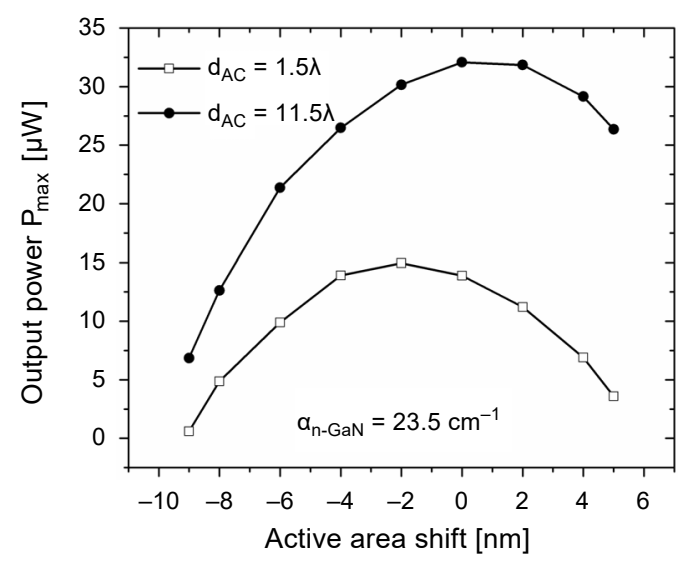

Fig. 8. Maximal output powers as functions of the active area shift, for two different positions of the active area in the resonator: $d_{\mathrm{AC}}=1.5 \lambda$ (active area near the DBR), and $d_{\mathrm{AC}}=11.5 \lambda$ (active area in the center of the resonator). 


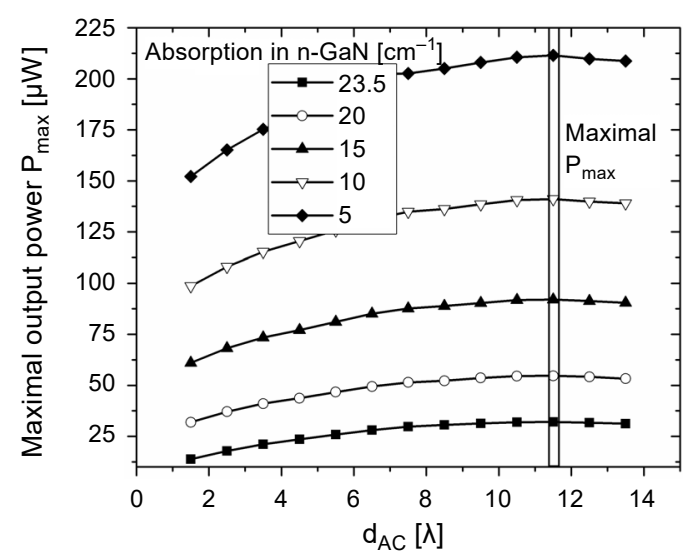

Fig. 9. Maximal output powers as functions of the distance from the bottom DBR to the active area, for different values of absorption in $\mathrm{n}-\mathrm{GaN}$ resonator layer.

of the resonator. For the laser with $d_{\mathrm{AC}}=11.5 \lambda$ the maximal temperature increase in the structure is $22 \%$ lower in comparison with the base structure with $d_{\mathrm{AC}}=1.5 \lambda$. For the VCSEL with the active area placed close to the bottom DBR $\left(d_{\mathrm{AC}}=1.5 \lambda\right)$, the heat is extracted from the active area mainly in the upward direction. When the active area is in the middle of the resonator $\left(d_{\mathrm{AC}}=11.5 \lambda\right)$, the heat is extracted at the same time in the upward and downward directions. This solution improves the thermal properties of the laser. Then the powers emitted by the lasers were calculated. Figure 8 shows maximal output powers as a function of the active area shift for two distances considered before: $d_{\mathrm{AC}}=11.5 \lambda$ and $d_{\mathrm{AC}}=1.5 \lambda$. The maximal output power is over two times higher, when the active area is placed at the center of the resonator $\left(d_{\mathrm{AC}}=11.5 \lambda\right)$ in comparison to the case where it is near the bottom $\operatorname{DBR}\left(d_{\mathrm{AC}}=1.5 \lambda\right)$.

Figure 9 shows the impact of the position of the active area on the maximal output power $P_{\max }$. In the same graph, different values of the absorption in the $\mathrm{n}-\mathrm{GaN}$ resonator layer are also considered. According to the results in Fig. 9, the highest output power is obtained for $d_{\mathrm{AC}}=11.5 \lambda$ for all the considered absorptions. The maximal output power depends strongly on the absorption in the $\mathrm{n}-\mathrm{GaN}$ layer. The difference between the two extreme cases $\left(\alpha_{\mathrm{n}-\mathrm{GaN}}=5\right.$ and $\left.23.5 \mathrm{~cm}^{-1}\right)$ is over 6 -fold.

\section{Conclusions}

According to our simulations, in the considered VCSEL, where the active area is located $1.5 \lambda$ from the bottom DBR, a detuning between the anti-node and the center of the active region within a $\pm 7-\mathrm{nm}$ range will not prevent the device from lasing in the case of relatively high internal loses. The impact of this detuning is less significant when the internal losses are lower.

In TJ nitride VCSELs, putting the active region at the center of the resonator can be considered. This solution improves the thermal properties of the structure which 
results in an over twofold increase of the emitted power, when compared with the standard configuration, where the active region is near the bottom DBR. Such a design, however, requires a good control of the position of the standing wave anti-nodes, which in nitride resonators can be a challenge.

Acknowledgements - This work was supported by the Polish National Science Centre (grant No. 2018/29/ N/ST7/02151) and the Polish Ministry of Science and Higher Education (grant No. I-3/501/17-3-1-758).

\section{References}

[1] Takaoka K., Ishikawa M., Hatakoshi G., Low-threshold and high-temperature operation of In GaAlP-based proton-implanted red VCSELs, IEEE Journal of Selected Topics in Quantum Electronics 7(2), 2001, pp. 381-385, DOI: 10.1109/2944.954154.

[2] Higuchi Y., Omae K., Matsumura H., Mukai T., Room-temperature CW lasing of a GaN-based vertical-cavity surface-emitting laser by current injection, Applied Physics Express 1(12), 2008, article 121102, DOI: 10.1143/APEX.1.121102.

[3] Lu T.C., KaO C.C., KuO H.C., HuAng G.S., WANG S.C., CW lasing of current injection blue GaN-based vertical cavity surface emitting laser, Applied Physics Letters 92(14), 2008, article 141102, DOI: $10.1063 / 1.2908034$.

[4] Forman C.A., Lee S., Young E.C., Kearns J.A., Cohen D.A., Leonard J.T., Margalith T., DenBaArs S.P., NAKamura S., Continuous-wave operation of m-plane GaN-based vertical-cavity surface-emitting lasers with a tunnel junction intracavity contact, Applied Physics Letters 112(11), 2018, article 111106, DOI: $10.1063 / 1.5007746$.

[5] Kearns J.A., Back J., Cohen D.A., DenBaars S.P., Nakamura S., Demonstration of blue semipolar (20기) GaN-based vertical-cavity surface-emitting lasers, Optics Express 27(17), 2019, pp. 23707 -23713, DOI: 10.1364/OE.27.023707.

[6] Greco G, Iucolano F, Roccaforte F., Ohmic contacts to gallium nitride materials, Applied Surface Science 383, 2016, pp. 324-345, DOI: 10.1016/j.apsusc.2016.04.016.

[7] Kuramoto M., Kobayashi S., Akagi T., Tazawa K., Tanaka K., Saito T., Takeuchi T., High-power GaN-based vertical-cavity surface-emitting lasers with AlInN/GaN distributed Bragg reflectors, Applied Sciences 9(3), 2019, article 416, DOI: 10.3390/app9030416.

[8] Sarzala R.P., NAKWASki W., Optimization of $1.3 \mu \mathrm{m}$ GaAs-based oxide-confined (GaIn)(NAs) vertical-cavity surface-emitting lasers for low-threshold room-temperature operation, Journal of Physics: Condensed Matter 16(31), 2004, pp. S3121-S3140, DOI: 10.1088/0953-8984/16/31/009.

[9] Kuc M., SarzaŁa R.P., NAKWASKi W., Thermal crosstalk in arrays of III-N-based lasers, Materials Science and Engineering: B 178(20), 2013, pp. 1395-1402, DOI: 10.1016/j.mseb.2013.08.013.

[10] SaRzala R.P., ŚPIEWAK P., WASIAK M., Influence of resonator length on performance of nitride TJ VCSEL, IEEE Journal of Quantum Electronics 55(6), 2019, article 2400509, DOI: 10.1109/ JQE.2019.2946386.

[11] Xu D., Tong C., Yoon S.F., Fan W., Zhang D.H., Wasiak M., Piskorski Ł., Gutowski K., Sarzala R.P., NAKWASKI W., Room-temperature continuous-wave operation of the In $(\mathrm{Ga}) \mathrm{As} / \mathrm{GaAs}$ quantum-dot VCSELs for the $1.3 \mu \mathrm{m}$ optical-fibre communication, Semiconductor Science and Technology 24(5), 2009, article 055003, DOI: 10.1088/0268-1242/24/5/055003.

[12] Wasiak M., Śpiewak P., Moser P., Walczak J., SarzaŁa R.P., Czyszanowski T., Lott J.A., Numerical model of capacitance in vertical-cavity surface-emitting lasers, Journal of Physics D: Applied Physics 49(17), 2016, article 175104, DOI: 10.1088/0022-3727/49/17/175104.

[13] SarzaŁa R.P., SokóŁ A.K., Kuc M., NAKwASKi W., How to enhance a room-temperature operation of diode lasers and their arrays, Optica Applicata 46(2), 2016, pp. 213 -226, DOI: 10.5277/oa160206. 
[14] Leonard J.T., Young E.C., Yonkee B.P., Cohen D.A., Margalith T., DenBaArs S.P., Speck J.S., NAKAMURA S., Demonstration of a III-nitride vertical-cavity surface-emitting laser with a III-nitride tunnel junction intracavity contact, Applied Physics Letters 107(9), 2015, article 091105, DOI: $10.1063 / 1.4929944$.

[15] Kuramoto M., Sasaoka C., Futagawa N., Nido M., Yamaguchi A.A., Reduction of internal loss and threshold current in a laser diode with a ridge by selective re-growth (RiS- $L D$ ), Physica Status Solidi (A) 192(2), 2002, pp. 329-334, DOI: 10.1002/1521-396X(200208)192:2\%3C329::AID-PS SA329\%3E3.0.CO;2-A.

[16] Perlin P., Czyszanowski T., Marona L., Grzanka S., Kafar A., Stanczyk S., Suski T., Leszczyński M., Boćkowski M., MuzioŁ G., Kuc M, SarzaŁa R.P., Highly doped GaN: a material for plasmonic claddings for blue/green In GaN laser diodes, Proceedings of SPIE 8262, 2012, article 826216, DOI: $10.1117 / 12.906866$.

[17] Hamaguchi T., Tanaka M., Mitomo J., Nakajima H., Ito M., Ohara M., Kobayashi N., Fuji K., Watanabe H., Satou S., Koda R., Narui H., Lateral optical confinement of GaN-based VCSEL using an atomically smooth monolithic curved mirror, Scientific Reports 8(1), 2018, article 10350, DOI: $10.1038 / \mathrm{s} 41598-018-28418-6$.

[18] Shen C.C., Lu Y.T., Yeh Y.W., Chen C.Y., Chen Y.T., Sher C.W., Lee P.T., Shih Y.H., Lu T.C., Wu T., Chiu C.H., Kuo H.C., Design and fabrication of the reliable GaN based vertical-cavity surface-emitting laser via tunnel junction, Crystals 9(4), 2019, article 187, DOI: 10.3390/cryst9040187.

Received October 16, 2019

in revised form January 27, 2020 